\title{
CASOS CLIINICOS
}
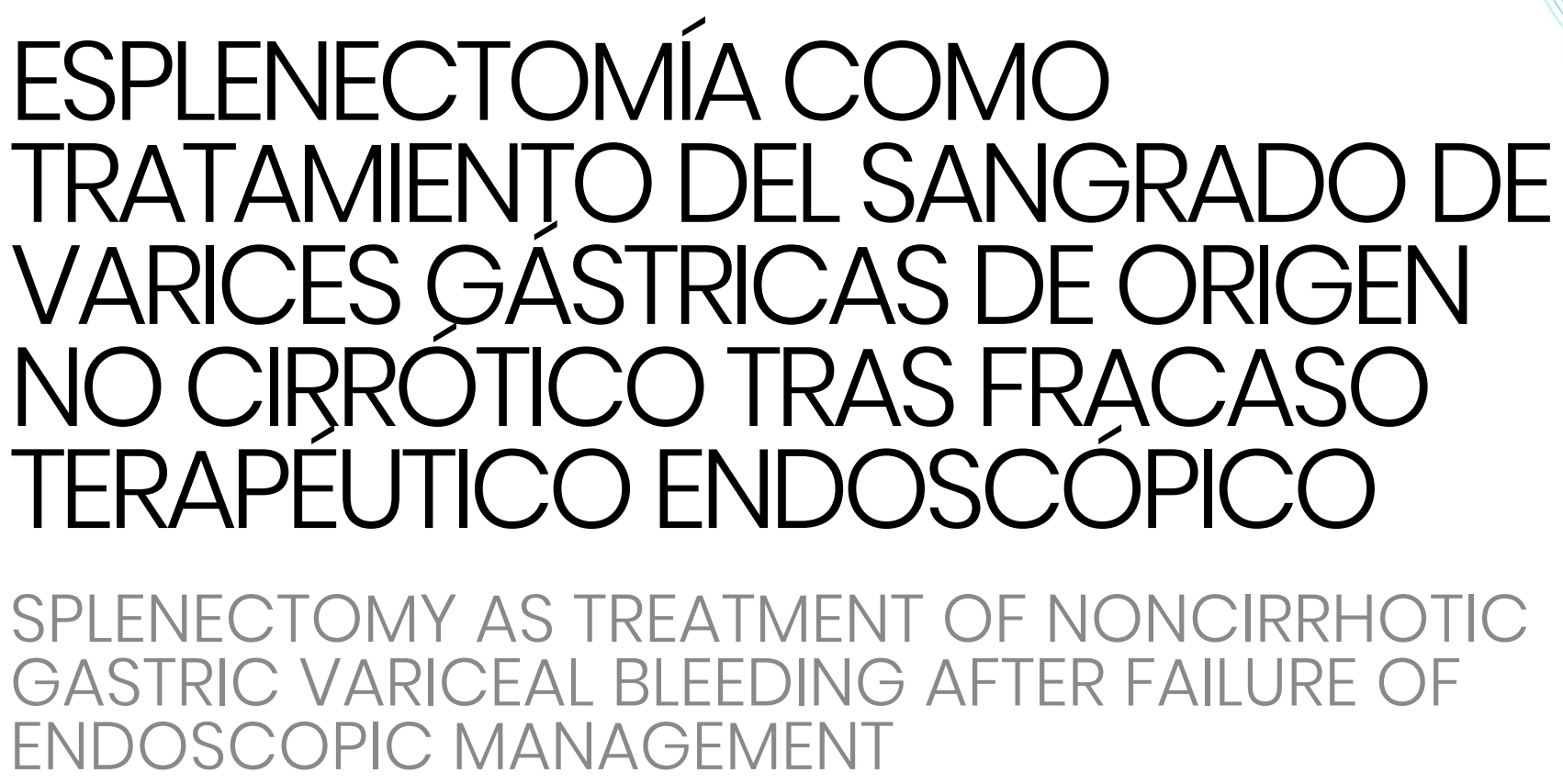

\section{Delgado Maroto A', Diéguez Castillo C', Lendínez Romero I²}

'UGC Aparato Digestivo. Hospital Torrecárdenas. Almería.

${ }^{2}$ UGC Cirugía General y Digestiva. Hospital Torrecárdenas

\section{Resumen}

El sangrado de varices gástricas por hipertensión portal izquierda es una complicación infrecuente, grave y de difícil control. Exponemos el caso de un paciente con sangrado de varices gástricas secundarias a la trombosis de la vena esplénica por pancreatitis aguda grave tratado de forma exitosa mediante esplenectomía urgente.

Palabras clave: varices gástricas, hipertensión portal, trombosis de vena esplénica.

\section{CORRESPONDENCIA}

Ana Delgado Maroto

Hospital Torrecárdenas

04009 Almería

anadmgr@gmail.com

Fecha de envío: 21/09/2020

Fecha de aceptación: 10/10/2020

\section{Abstract}

Gastric variceal bleeding caused by left-sided portal hypertension is a infrequent, severe condition, which is very difficult to control. We report the successful management by urgent splenectomy of a case of gastric variceal bleeding secondary to splenic vein thrombosis pancreatitis-induced. thrombosis.

Keywords: gastric varices, portal hypertension, splenic vein

\section{Introducción}

La hipertensión portal de origen no cirrótico, también llamada hipertensión portal izquierda o segmentaria, se caracteriza por la presencia de varices gástricas aisladas y esplenomegalia junto con una función hepática normal ${ }^{1}$. Las varices gástricas son más comunes en hipertensión portal de origen no cirrótico. Pueden deberse a diferentes causas siendo la principal la patología pancreática. Dentro de esta, la pancreatitis crónica es la causa más común, aunque también 
se han descrito casos asociados a neoplasias pancreáticas, pancreatitis aguda y pseudoquistes pancreáticos..$^{2,3} \mathrm{El}$ contacto estrecho de la vena esplénica con el páncreas la hace susceptible a oclusión/ trombosis en caso de inflamación, produciéndose una redirección del flujo sanguíneo a través de los vasos gástricos posteriores y vasos cortos a las venas coronarias que drenan en la vena porta y, a través de las venas gastroepiploicas, a la vena mesentérica superior. De esta forma, el retículo venoso submucoso del fundus gástrico representa un puente anatómico entre las venas gástricas cortas y posteriores con las venas coronarias que, como consecuencia del aumento de la presión, puede dilatarse formando varices. ${ }^{3,4}$

\section{Caso Clínico}

Presentamos el caso de un varón de 63 años con antecedente de pancreatitis aguda grave complicada hace más de 10 años, que desarrolla hipertensión portal secundaria a trombosis de vena mesentérica y cavernomatosis portal (Figura 1). Presenta varices esofágicas y fúndicas, esplenomegalia y una función hepática normal, estando en tratamiento con profilaxis primaria del sangrado con propranolol. Acude a urgencias por cuadro de hematemesis con repercusión hemodinámica, con hipotensión arterial (90/50 $\mathrm{mmHg}$ ) y taquicardia (105 lpm). En la analítica al ingreso destaca urea $74 \mathrm{mg} / \mathrm{dL}$, creatinina $1 \mathrm{mg} / \mathrm{dL}$, función hepática normal, anemia aguda con hemoglobina $8 \mathrm{~g} / \mathrm{dL}$, plaquetas $85.000 / \mu \mathrm{L}$ y coagulación normal. Realizamos endoscopia digestiva alta objetivándose varices esofágicas grandes sin estigmas de sangrado reciente y sangrado activo procedente de varices fúndicas sin conseguir un correcto manejo endoscópico. El paciente sufre un empeoramiento clínico, con sangrado activo e hipotensión persistente a pesar de fluidoterapia y transfusión de hemoderivados. Ante persistencia del sangrado se opta por esplenectomía urgente objetivándose esplenomegalia con múltiples adherencias en tejido circundante, vasos cortos ingurgitados y dilatados y abundante colateralidad periesplénica. Se procede a liberación de adherencias, esplenectomía y sección de vasos cortos sin incidencias. Posteriormente el paciente evoluciona de forma favorable, con cese del sangrado y sin complicaciones postoperatorias. Tres semanas después realizamos control endoscópico que muestra disminución del tamaño de varices fúndicas e incluimos al paciente en programa de ligadura endoscópica de varices esofágicas.

\section{Discusión}

El sangrado de varices gástricas es una complicación grave de difícil control. Supone menos del $1 \%$ de causas de sangrado alto, aunque con una mortalidad que puede alcanzar el $20 \%$ en 6 semanas. El riesgo de mortalidad por varices gástricas secundarias a hipertensión portal izquierda es mayor que al sangrado por varices de otras etiologías. ${ }^{3}$ El manejo endoscópico mediante escleroterapia ha demostrado una alta tasa de fallo para el control agudo del sangrado y de resangrado precoz. Por ello, la opción terapéutica más recomendada en caso de hipertensión portal izquierda sintomática es la esplenectomía y corrección de la causa primaria si es posible. El objetivo es reducir la presión del sistema mediante la interrupción del flujo de la arteria esplénica y consecuentemente del drenaje venoso y las varices fúndicas. ${ }^{4,5}$ En el caso de nuestro paciente, presenta un sangrado activo procedente de varices gástricas y, a su vez, presenta varices esofágicas grandes secundarias a cavernomatosis portal. Se

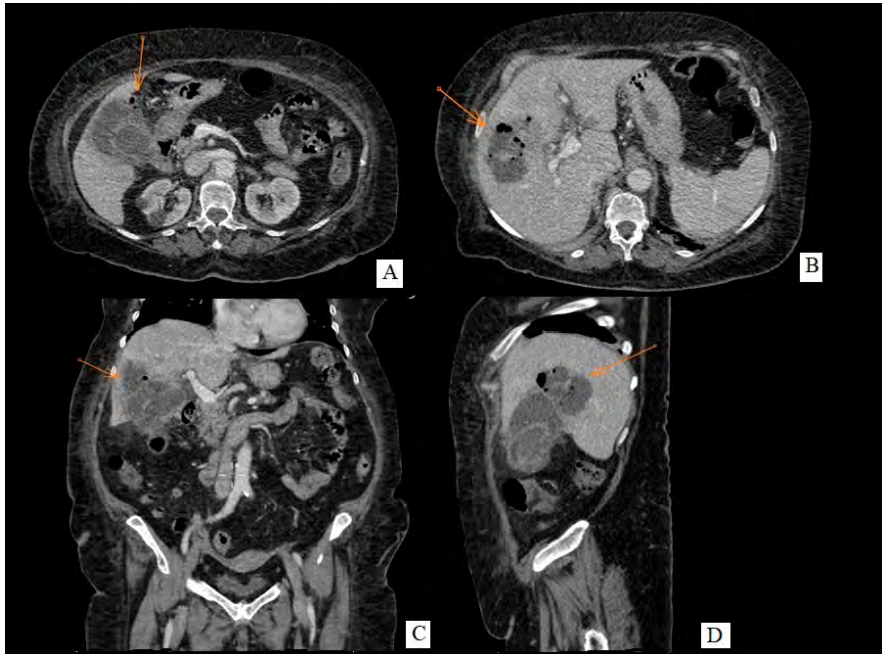

Figura 1

TCABDOMINAL: varices fúndicas y circulación colateral peripancreática.

decide realización de esplenectomía para descomprimir el territorio venoso ya que la mayoría de las varices del paciente dependen del territorio periesplénico y estas son las causantes del sangrado agudo, posponiéndose el tratamiento de las varices esofágicas mediante ligadura endoscópica a un segundo tiempo. Otras opciones terapéuticas como la embolización de la arteria esplénica deberían reservarse en caso de sangrado activo en el que no pueda realizarse esplenectomía. ${ }^{2}$ Actualmente no se aconseja realizar la esplenectomía como prevención primaria en pacientes con hipertensión portal izquierda no complicada. ${ }^{4}$

\section{Bibliografía}

1. Franco-Avilés, L; Hernández-Rocha, FI; Mercado, U; Malvido-Torres, CG. Hipertensión portal segmentaria por trombosis de la vena esplénica causada por pancreatitis. Rev Med Inst Mex Seguro Soc. 2017;55(6):788-90

2. Kokabi N, Lee E, Echevarria Cet al. Sinistral portal hypertension: presentation, radiological findings, and treatment options - a case report. J Radiol Case Rep 2010; 4(10):14-20

3. Tang SH, Zeng WZ, He QW, et al. Repeated pancreatitis-induced splenic vein thrombosis leads to intractable gastric variceal bleeding: a case report and review. World J Clin Cases 2015;3:920-5

4. Patrono D, Benvenga R, Moro F et al. Left-sided portal hipertension: Successful management by laparoscopic splenectomy following splenic artery embolization. Int J Surg Case Rep 2014; 5(10): 652-5.

5. Menasherian-Yaccobe, L.; Jaqua, N.T.; Kenny, P. Successful treatment of bleeding gastric varices with splenectomy in a patient with splenic, portal, and mesenteric thromboses. Case Rep. Surg 2013, 2013, 273531 